\title{
Inhibition and facilitation of eating by electric shock: III. A further study of the role of strain and of shock level'
}

\author{
Graham M. Sterritt \\ UNIVERSITY OF COLORADO MEDICAL CENTER
}

\begin{abstract}
Abstraet
Wistar and Long-Evans rats were given brief shocks while eating. No level of shock intensity led to net overeating by comparison to unshocked controls, although several shock intensities led to reliable overeating during shock. Shock had somewhat less effect on eating in Wistars than in Long-Evans rats.
\end{abstract}

\section{Problem}

In a pilot study of handling as a variable affecting eating behavior in rats that get brief electric shocks to the paws during free eating periods, there was little effect associated with handling, but some suggestion that shock of $0.15 \mathrm{ma}$ intensity might lead to reliable net overeating in rats given shock, by comparison to unshocked rats. In a still earlier experiment (Sterritt \& Shemberg, 1963) $0.1 \mathrm{ma}$ had yielded little inhibition or facilitation of eating, while $0.2 \mathrm{ma}$ had led to substantial overeating during shock a n d inhibition between shocks, with net inhibition of eating by comparison to unshocked Ss. It appeared possible that 0.15 ma might represent an optimal shock intensity, high enough to facilitate eating and low enough so as not to inhibit eating between shocks.

Wistar albino rats are reputed to be more emotional than other strains. If so, they might be more responsive in the shock-eating situation than either of the strains investigated previously.

With these corsiderations in mind, Wistar albinos were compared with Long-Evans hooded rats under eight shock levels, representing fine gradations in the range of intensites that held the greatest promise of yielding reliable net overeating as a response to shock.

\section{Subjects}

The Ss were 61 Wistar albino rats, obtained from Charles River Breeding Labs, Brookline, Mass., and Animal Supply Co., Napa, California and 59 Long-Evans hooded rats obtained from Rockland Farms, New York City, N.Y., and Diablo Labs, Berkeley, Calif。

\section{Apparatus}

A programming apparatus was designed to make E's task easier (Sterritt, 1964). Shock was delivered automatically and $\mathrm{E}$ had only to watch the rat and make a mark in that column on the data sheet that was illuminated at the moment a pellet was taken. Accuracy of shock delivery and reliability of scoring of pellettaking were improved by this innovation.

The stepping switch in this apparatus had an 11-step cycle, making it convenient to give shock during the first $5 \mathrm{sec}$. of every $55 \mathrm{sec}$., instead of every $60 \mathrm{sec}$. as in prior experiments.

In all other respects, the apparatus was the same as that used previously (Sterritt \& Shemberg, 1963).

\section{Procedure}

Ss were put on a limited feeding schedule, reducing body weight by $20 \%$ before the single $20 \mathrm{~min}$. familiarization period, in which food, but no shock, was given in the apparatus.

On the following day the 4-day "deprived phase" began. Shock was given, except to the No Shock Group, during the first 5 sec. of every 55 sec. interval. Shock was not given during the first 55 sec., when $\mathrm{S}$ was assumed to be "warming up." Each S remained in the apparatus for 21 consecutive $55 \mathrm{sec}$. intervals each day, then went into a feeder cage to receive the balance of the daily feeding, maintaining body weight at $80 \%$ of normal. Water was available ad $1 \mathrm{ib}$ at. all times except in the apparatus.

Following the "deprived phase," each $\mathrm{S}$ was given food ad $1 \mathrm{ib}$ in the home cage. Ss were placed in the apparatus each day, with shock as before, for the four days following the deprived phase, but during this "sated phase" they were never deprived of food and were further sated with wet mash just before entering the apparatus.

\section{Results}

Figure 1 shows eating during shock and total eating for Wistar and for Long-Evans rats during the deprived phase. At no shock intensity, in either strain, did shocked rats consume reliably greater total amounts than were eaten by the No Shock Group. Among the hooded rats the mean total amounts consumed at $0.10,0.15$ and $0.20 \mathrm{ma}$ of shock all appear to exceed the mean total amount eaten by the No Shock Group; however, when the data from these three shock groups are tested statistically against the No Shock Group data, taking one shocked group at a time or pooling the three together, the differences do not in any case approach conventional significance levels.

The results with the Long-Evans rats of this study resemble those obtained in previous experiments with this strain. These Ss show great overeating during shock at moderate intensities of shock (Long-Evans rats, 


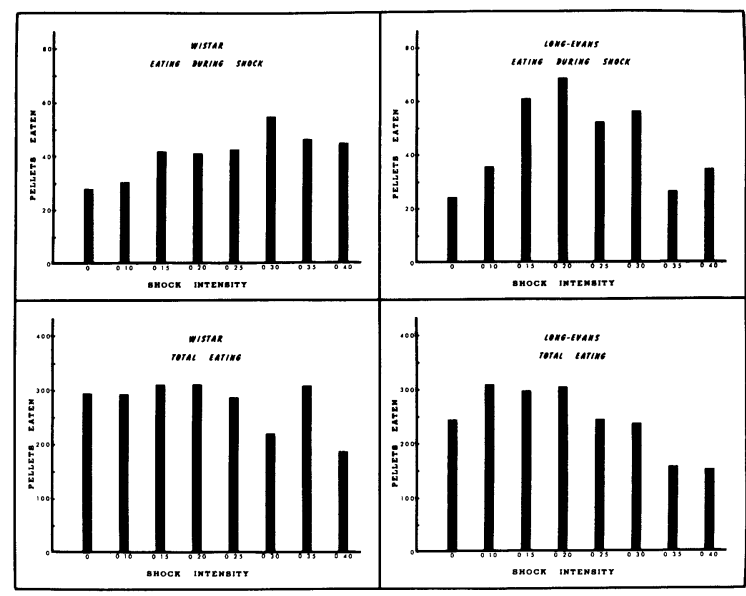

Fig. 1. Mean numbers of pellets eaten during shock and in toto by Wistar and by Long-Evans rats.

Kruskal-Wallace test for overall differences between shock level groups in eating during shock, $\mathrm{p}<.01$; the No Shock Group is exceeded by the $0.10,0.15,0.20$, 0.25 and 0.30 groups; in each case the Mann Whitney $\mathrm{U}$ test is signficant at $\mathrm{p}=.01$ or better-a non-parametric test was used because of reliable differences between groups in variance). At higher intensities, on the other hand, shock does not facilitate eating during shock, but does inhibit eating between shocks (one-way analysis of variance based on eating between shocks, overall F test, $\mathrm{p}<.01$; No Shock Group vs. 0.35 and $0.40 \mathrm{ma}$ Shock Groups, in each case $\mathrm{p}<.01$ )。

The Wistar albino rats did not eat reliably more during shock, in absolute numbers of pellets, by comparison to the No Shock Group. However, the percentage taken during the shock-on time is greater within the $0.15,0.20,0.25$ and 0.40 shock groups than in the No Shock Group; in each case the Mann Whitney $U$ is significant at $p<.05$ or better.

The Wistars resemble the Long-Evans Ss in showing reliable inhibition of eating between shocks at higher shock intensities (Wistars, eating between shocks, oneway analysis of variance, overall $F$ test, $p=.05$; No Shock Group vs. 0.40 ma Shock Group, $\mathrm{p}<.01$ ).

As in previous studies, rates of eating were more variable during the sated phase and no significant differences were found then.

\section{Diseussion}

The absence of net overeating in either strain, at any shock intensity, confirms the findings of earlier experiments (Sterritt, 1962; Sterritt \& Shemberg, 1963) supporting the conclusion that shocking hungry rats during free eating periods is not an effective means of inducing true overeating.

Wistar albinos are, if anything, le s s affected by shock than Long-Evans hooded rats. The rate of eating duiring shock rises rapidly as shock intensity increases in Long-Evans Ss, while eating during shock is less affected at moderate shock intensites in Wistars.

\section{References}

STERRITT, G. M. Inhibition and facilitation of eating by electric shock. J. comp. physiol. Psychol., 1962, 55, 226-229.

STERRITT, G. M., \& SHEMBERG, K. Inhibition and facilitation of eating by electric shock: II. Shock level, shock schedule and strain of rats. J. psychosom. Res., 1963, 7, 215-222.

STERRITT, G. M. A device to aid in the observation of behavior as a function of time intervals. Percept. mot. Skills, 1964, $18,116$.

\section{Note}

1. Supported by a research grant (M-2776) from the National Institute of Mental Health, and a grant from the Fluid Research Fund of the University of Colorado Medical Center. The author is at present the recipient of a Research Career Development Award from the National Institute of Child Health and Human Development. 